\title{
Pubblicità e memoria mediale: appunti in itinere*
}

\author{
Cinzia Bianchi \\ Università degli Studi di Modena e Reggio Emilia \\ e-mail: cinzia.bianchi@unimore.it \\ web: www.cinziabianchi.it
}

\begin{abstract}
The essay aims at relating the outcomes of a multidisciplinary research project focused on the relationship between a specific kind of social discourse, such as advertising, and the concept of "media memory". Through the analysis of concrete examples of commercials, we have tried to outline a composite field of studies ranging from semiotic studies on individual and cultural memory, to the role mass media play in defining a collective memory, up to the processes of interpretation that the reader/user activates to understand texts which create a network of references to our past.
\end{abstract}

\section{Parole chiave}

Pubblicità, memoria mediale, Eco, Lotman

\section{Key Words}

Advertising, media memory, Eco, Lotman

\section{Sommario}

1. Introduzione: memoria mediale e media studies

2. E la pubblicità?

3. Cultura e memoria secondo Lotman ed Eco

4. Esempi di analisi: pubblicità e pratiche di replicabilità

5 . Note conclusive

Bibliografia

* Questo saggio, scritto nella sua prima versione nel 2013, ha avuto diverse revisioni bibliografiche a causa del prolungarsi dei tempi di pubblicazione. Si tratta di un saggio introduttivo all'argomento, pensato nella sua prima stesura per gli studiosi di linguistica. Ne pubblichiamo qui una versione parziale senza analisi semiotiche specifiche, certi che comunque l'argomento meriti di essere portato all'attenzione anche degli studiosi di semiotica, alcuni dei quali ne conoscono già gli sviluppi più recenti. 


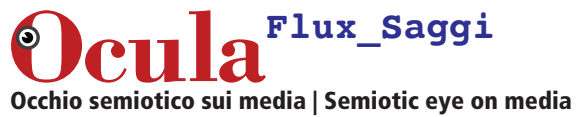

Cinzia Bianchi | Pubblicità e memoria mediale: appunti in itinere

\section{Introduzione: memoria mediale e media studies}

Partiamo dal titolo di questo saggio che coinvolge già nella sua semplice enunciazione due elementi: la pubblicità come genere specifico del discorso sociale, e il concetto di memoria, molto discusso negli ultimi anni in ambiti disciplinari diversi, tra cui anche all'interno degli studi semiotici (cfr. in particolare Demaria 2006). Sempre l'analisi del titolo ci indica poi che non si parlerà del concetto di memoria nella sua generalità e ampiezza teorica, ma di "memoria mediale", volendo restringere il campo di riflessione a un ambito più consono alla cultura di massa, in cui i media sono i protagonisti indiscussi ${ }^{1}$.

Ciò nonostante, i possibili svolgimenti del nostro argomenti sono perlomeno due, molto collegati tra loro. Potremmo soffermarci sulla memoria della pubblicità, cioè ricostruire storicamente l'evoluzione di un genere specifico degli annunci-stampa e dell'audiovisivo pubblicitario nell'ambito dei fenomeni di consumo. Oppure potremmo seguire una riflessione sui modi attraverso cui è possibile costruire (e/o ricostruire) una memoria collettiva (cioè più o meno condivisa culturalmente) attraverso varie fonti audiovisive tra cui quella pubblicitaria.

Il primo svolgimento possibile, all'intersezione tra storia della pubblicità e studi culturali, mette in gioco alcune questioni pratiche (ma anche teoriche) di consultazione e, ancora più a monte, di archiviazione/selezione del materiale audiovisivo. Argomento particolarmente sentito e discusso nell'ambito dei media studies in quei lavori che riguardano la televisione, la cosiddetta "televisione della memoria" (fiction storiche, documentari di eventi del passato ma anche più recenti forme narrative come le docufiction), una televisione che in ogni caso si percepisce come debitrice a sua volta degli studi sul cinema (cfr. in particolare Bianchi 2008).

Non si tratta quindi di un argomento nuovo nel panorama degli studi sull'audiovisivo che da molti anni ormai hanno affrontato temi come: 1) l'importanza della fonte audiovisiva per la ricerca storica e i problemi legati alla sua archiviazione (e consultazione); 2) la scelta inevitabile che si deve fare per la conservazione, discernendo, per esempio, nel flusso dell'informazione tra ciò che è evento degno di essere conservato e ciò che non lo è; 3) le possibili conseguenze per la memoria collettiva della sovrabbondanza di narrazioni storiche per immagini in movimento (cfr. in particolare Grasso 2006).

1 Come ci ricorda Roger Silverstone (1999) i media (cinema, radio, televisione e ormai anche Internet) hanno la «capacità sia di creare e mantenere un ordine nella vita quotidiana, sia di trovare una posizione all'interno di questo ordine» (Silvestrone 1999 [2002: 183]) contribuendo alla formazione del senso comune alla base del legame sociale su cui si fonda la memoria sociale. 


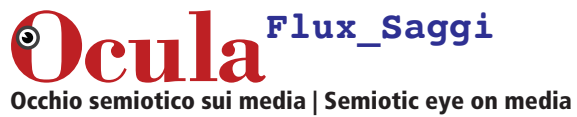

Cinzia Bianchi | Pubblicità e memoria mediale: appunti in itinere

Il primo tema, quello della televisione come fonte di ricerca storica, è molto dibattuto. L'idea che l'immagine televisiva possa essere fonte documentale sembra prevalere: gli archivi televisivi conservano immagini del passato e gli storici, a volte a malincuore e senza perdere occasione di sottolinearne le insidie (cfr. Bourdon in Grasso 2006), devono considerarle come uno dei possibili documenti della loro ricerca.

Il valore documentale dell'immagine audiovisiva dipende anche dal fatto che la televisione è divenuta un mezzo inevitabile del dispiegarsi stesso della storia, sia come agente attivo della Grande Storia sia della storia più contingente e quotidiana, delle diverse storie individuali e collettive. Sembra infatti essere pacifico che la fonte audiovisiva sia predominante nella nostra quotidiana necessità di informazione e approfondimento che avviene, aggiungiamo noi, attraverso media più o meno tradizionali, dalla televisione ai new media.

Dobbiamo arrivare al terzo tema indicato per vedere dispiegarsi davanti a noi collegamenti con la sopracitata costruzione e ricostruzione di una memoria collettiva.

I media in generale sono veri e propri luoghi di sintesi di punti di vista individuali e collettivi sul passato, di cultura 'alta' e 'bassa', popolare ed elitaria. Come ci ricorda Roger Silverstone,

non esiste distinzione inequivocabile fra la rappresentazione storica e la rappresentazione popolare del passato: sono fuse insieme e stanno in competizione nello spazio pubblico, insieme per noi definiscono testi e contesti, per l'identità, la comunità e, cosa forse più importante che le comprende entrambe, per la fede e l'azione (Silverstone 1999 [2002: 201]).

L'immagine televisiva in particolare può poi essere terreno di coagulazione di una memoria condivisa che diventa tale perché viene raccontata e per questo si colloca all'interno del circuito culturale. Il nostro stesso passato personale è legato a quello di immagini mediate, e la stessa televisione celebra continuamente il proprio passato e al contempo, attraverso la produzione di fiction e documentari, commemora il passato nazionale, contribuendo a riscrivere una storia pubblica, e con essa la struttura connettiva della nostra memoria culturale. Seguendo questo ragionamento sembra andarsi ad attenuare la distinzione tra discorso storico e memoria del passato su cui molti studiosi hanno riflettuto (Ricoeur 1998; Demaria 2006): il racconto storico e la memoria del passato contribuiscono entrambi alla costruzione dell'identità e della memoria collettiva; sono entrambi fonte di ispirazione per molta televisione, la quale, a sua volta, contribuisce a rafforzarne e definirne i confini. Come sottolinea Carini: "il piccolo schermo racconta il nostro passato, selezionando le immagini, attribuendo loro una gerarchia, stabilendo tempi e modalità di lettura" (Carini, in Grasso 2006: 85); è questo un processo complesso di rammemorazione o commemorazione in cui la produzione televisiva, operando grandi semplificazioni, interagisce con altri generi discorsivi, come, per esempio diari, monumenti, canzoni, film. Si tratta di una quantità rilevante, forse sovrabbondante e variegata di narrazioni, a cui ci permettiamo di aggiungere anche quella specifica della pubblicità. 


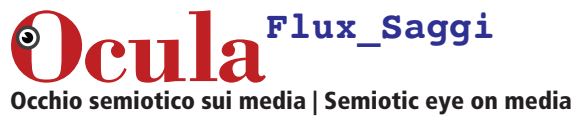

Cinzia Bianchi | Pubblicità e memoria mediale: appunti in itinere

\section{E la pubblicità?}

Va preliminarmente sottolineato come nell'ambito di questo composito campo di studi si trovano solo sporadici accenni alla questione dell'archiviazione e della memoria degli spot pubblicitari, di cui si riconosce l'importanza in senso molto generale ma di cui al contempo si intuisce la difficoltà per una loro organica archiviazione e conservazione.

Teniamo presente che non dobbiamo sovrapporre troppo le questioni dell'archiviazione del materiale audiovisivo con le questioni della memoria collettiva: l'archivio è solo memoria istituzionale (o meglio dell'istituzione che segue propri criteri di selezione, conservazione e diffusione del materiale archiviato). Ma è anche vero che la questione della praticabilità di una conservazione integrale riguarda in specifico l'audiovisivo pubblicitario. Ci si chiede: tutti gli spot pubblicitari vanno conservati? A domanda così generale c'è una risposta pressoché unanime perché gli spot rilevano molto della nostra società dei consumi e del fascino di alcuni prodotti in un dato periodo. Di fatto poi, almeno in Italia, l'archiviazione di questi audiovisivi è ancora più difficoltosa che altrove. Faccio solo l'esempio dell'organizzazione delle Teche RAI, operazione ragguardevole perché permette una consultazione più semplice dell’immenso patrimonio audiovisivo della televisione di stato. Ciò nonostante, nella nuova archiviazione viene sacrificato completamente tutto il flusso televisivo, e in specifico appunto la pubblicità nelle sue variegate forme (spot, promo, lancio dei programmi, ecc.) Ricordiamo come alcuni studiosi, come Guido Ferraro abbiano evidenziato come, nell'epoca di televisione composita, ibridata, di programmi sempre meno riconducibili a generi pre-definiti, ma progettati come una sorta di mix genetico, di "ricomposizione in forme miscelate e infinitamente variate" (Ferraro 2000: 23), la pubblicità possa essere considera in prospettiva sociosemiotica l'unica vera costante ritmica del flusso televisivo: indipendentemente dal programma trasmesso, l'interruzione pubblicitaria è nella televisione generalista l'unica costante che uno spettatore si può aspettare. $\mathrm{E}$ sappiamo quanto sia importante nella costruzione dell'abitudine di consumo tutto ciò che riguarda ritmo o ripetizione. Un'archiviazione per programmi comporta una sorta di cancellazione di una fonte di futura ricerca sull'audiovisivo $^{2}$, che però, dobbiamo dire, è ampiamente controbilanciata dai materiali presenti in Internet e dal 2005 in Youtube, ormai fonte quasi primaria, anche se spesso di bassa qualità, di forme brevi della comunicazione ${ }^{3}$, sia della nostra contemporaneità che del passato.

La frammentarietà dell'archiviazione e della sua conseguente fruizione è un dato peculiare della comunicazione pubblicitaria. Non per questo riteniamo che quest'ultima possa essere considerata meno rilevante di altre forme narrative nella costruzione della nostra memoria collettiva.

2 Sulla possibile valorizzazione del patrimonio audiovisivo in generale, cfr. anche Treleani 2014. Nel libro si riflette sui vantaggi della digitalizzazione e della messa on line degli archivi audiovisivi, ma anche sui rischi di alterazione del significato generale dei documenti così archiviati, specialmente per le future ricerche.

3 Cfr. in particolare Pezzini 2002, dove viene fornita una variegata casistica di 'forme brevi della comunicazione', dagli spot ai trailer, dai videoclip ai banner. 
Cinzia Bianchi | Pubblicità e memoria mediale: appunti in itinere

\section{Cultura e memoria secondo Lotman ed Eco}

Se volessimo indirizzare il nostro argomento verso un percorso più filosofico e semiotico, dovremmo citare sicuramente due insigni pensatori come Jury Lotman e Umberto Eco.

Lotman (cfr. in particolare Lotman-Uspenski 1975 e Lotman 1985) collega strettamente cultura e memoria. La cultura è memoria nel senso che è la registrazione, nella memoria, di quanto è già stato vissuto dalla collettività. $\mathrm{Al}$ momento del suo apparire una cultura non può essere riconosciuta come tale: di essa si acquista piena coscienza solo, per così dire, post factum. La cultura, per essere tale, ha bisogno della memoria, vale a dire della sua stessa 'registrazione' e rappresentazione. Nel descrivere tale processo, Lotman individua tre modi per assegnare un contenuto alla cultura, che può avvenire: a) attraverso un aumento quantitativo delle conoscenze; $\mathrm{b}$ ) attraverso una redistribuzione - e quindi attraverso una valutazione gerarchica di quel che è registrato nella memoria, che ha come conseguenza una continua riorganizzazione del sistema codificante; c) attraverso la dimenticanza - solo alcuni eventi vengono infatti tradotti in elementi testuali e poiché, per Lotman, ogni testo contribuisce sia alla memorizzazione che alla dimenticanza, il prevalere di uno dei due poli non può che dipendere dalle norme semiotiche di una data cultura 4 .

L'ampia prospettiva lotmaniana può essere integrata, a nostro avviso, con il contributo di Umberto Eco, il quale ci fornisce sia una riflessione generale sulla cultura sia gli strumenti teorici necessari per capirne trame e dinamiche. $\mathrm{E}$ in questo contesto è fondamentale il concetto di Enciclopedia.

L'Enciclopedia è presentata da Eco in Semiotica e filosofia del linguaggio come "l'insieme registrato di tutte le interpretazioni", la "libreria delle librerie", la "competenza globale" (Eco 1984: 109). È questa una definizione di semiotica generale che fa corrispondere l'Enciclopedia alla cultura in quanto tale, con le sue reticolari interconnessioni che contemplano anche interpretazioni contraddittorie e opposte segmentazioni del continuum. Ma ciò che più interessa il nostro discorso riguarda l'aspetto sociosemiotico del concetto di Enciclopedia: essa è da questo punto di vista la riserva di possibili interpretazioni a partire dalle quali il destinatario del testo seleziona quella più indicata e in tale processo si possono evidenziare i diversi livelli di possesso dell'Enciclopedia stessa. In ogni attività interpretativa, sostiene Eco, l’interprete è infatti

4 Non possiamo in questa sede approfondire il rapporto tra memoria e dimenticanza. Vogliamo però menzionare il lavoro di Elena Esposito, La memoria sociale (2001) in cui viene approfondito il rapporto tra media e tecniche di memorizzazione. Esposito ricorda come con la comparsa della stampa, e in particolare a partire XVII secolo, la memoria abbia iniziato a fondarsi sulla comunicazione e sui testi che non solo rendono la memoria archiviabile e disponibile, ma contengono e conservano le condizioni, le norme e le regole per la sua stessa testualizzazione. A partire da questo momento storico, e sempre più, nella forma della memoria (ricordare/dimenticare) il primato passa alla dimenticanza. La potenza della memoria è tanto maggiore quanto più consente di dimenticare, cioè di prescindere dalla conservazione di oggetti e dati specifici senza che essi vadano persi definitivamente. L'autrice sottolinea infine come le moderne 'tecnologie della memoria' si distinguano radicalmente da quelle precedenti perché non servono a mantenere direttamente i contenuti (a ricordare), ma soltanto a stabilire dei rinvii e dei collegamenti intertestuali. 


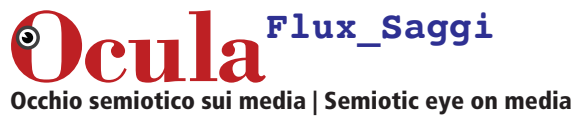

Cinzia Bianchi | Pubblicità e memoria mediale: appunti in itinere

tenuto a conoscere primariamente quella porzione di Enciclopedia necessaria a comprendere un dato testo. Si tratta di una competenza parziale che dipende da vari condizionamenti, ma soprattutto da quelli provenienti dalla cultura di riferimento. In questo senso le enciclopedie parziali, seppur proprie di gruppi, classi, etnie, devono essere considerate come partizioni o frammentazione dell'Enciclopedia Globale; fanno parte di un patrimonio comune in quanto repertorio dei saperi socialmente e culturalmente dati in un preciso periodo storico da un particolare gruppo. Ricostruire le trame di una porzione dell'Enciclopedia di un individuo, di una data comunità, di una data cultura è una vera e propria pratica di rammemorazione. E per procedere in questo tipo di analisi non possiamo fare a meno degli strumenti narratologici e di pragmatica del testo su cui Eco ci ha chiamato a riflettere nell'arco di almeno una ventina di anni, discutendo nei suoi libri di casi di coerenza testuale, di fabula e intreccio, di topic, di riferimento a un insieme di conoscenze presupposte da un qualsiasi testo, di implicatura conversazionale e testuale, di interpretazione ed eccessi interpretativi, e così via ${ }^{5}$.

Sono questi meccanismi narratologici e di pragmatica della lettura che attengono all'interpretazione testuale nella sua generalità, ma che ci saranno estremamente utili anche per perseguire il nostro obiettivo, cioè comprendere maggiormente come la pubblicità, in quanto forma testuale della nostra cultura, possa, attraverso operazioni semiotiche di citazione, imitazione, prestito e assimilazione, costruire le proprie reti di richiami al passato, ripetizioni variate e intertestualità che il fruitore, attingendo all'enciclopedia comune, può comprendere attraverso un suo peculiare processo interpretativo.

\section{Esempi di analisi: pubblicità e pratiche di replicabilità}

Conviene a questo punto della nostra argomentazione addentrarsi nella specificità della comunicazione pubblicitaria.

Ci sembrano particolarmente interessanti per il nostro discorso quei testi pubblicitari che, oltre a essere narrativamente efficaci a innescare un processo di interpretazione testuale nella sua generalità, ne sfruttano tutti i meccanismi più raffinati per produrre testi particolarmente accattivanti. Ci riferiamo a pubblicità non solo genericamente rammemorative, ma soprattutto commemorative - di anniversari specifici della marca, dell'azienda, del prodotto come, per esempio, i 50 anni del Cornetto Algida o della Fiat 500, i 132 anni della Barilla o i 130 della Edison, e così via ${ }^{6}$. Sono cioè quelle pubblicità che, consapevolmente, prendono in carico e sfruttano ottimamente la peculiarità di buona parte della cultura di massa contemporanea in quanto diffusa pratica di replicabilità.

5 Cfr. in particolare Eco 1979, 1990 e 1994 anche se possiamo trovare riflessioni sulle dinamiche interpretative in tutta la sua opera, almeno a partire dal Lector in fabula del 1979.

$6 \mathrm{Si}$ possono visionare gli spot analizzati in questo saggio al seguente indirizzo web: http://www.cinziabianchi.it/mm/av/memoria/. Si ricorda inoltre che una ricerca su Youtube potrebbe mostrare anche ulteriori filmati connessi, comprese molte parodie e rivisitazioni. 


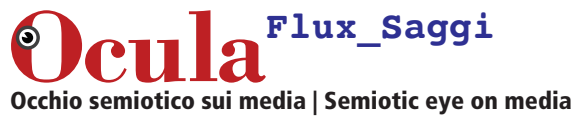

Cinzia Bianchi | Pubblicità e memoria mediale: appunti in itinere

Come ci ricordano Nicola Dusi e Lucio Spaziante nel libro Remix-Remake, "le pratiche di replicabilità consistono in procedure condivise di invenzione, originate partendo da memorie e archivi testuali e incorporando le prassi sedimentate di produzione e fruizione all'interno dei testi stessi" (2006: 10). E ancora:

se consideriamo il panorama attuale delle mutazioni, trasformazioni, contaminazioni audiovisive e musicali, potremmo partire dalla considerazione che nelle forme di replicabilità siamo di fronte sia a valorizzazioni dei testi assunti a testo di partenza (testo source) da parte dei testi di arrivo (testi target), sia all'uso di questi testi-fonte come sorta di pretesti da riaprire, rielaborare, riadattare. Il fenomeno del remake diventa così parte di una più generale forma contemporanea del mito, o di mitopoiesi (Dusi et al. 2006: 14)

Questi due passaggi dovrebbero rendere chiara la procedura attraverso cui si mettono in atto alcuni processi di valorizzazione nella dinamica testo source/testo target che può riguardare aspetti diversi dell'organizzazione testuale: la storia narrata, innanzitutto, ma spesso anche il comune riferimento culturale fino alla costruzione di un vero e proprio 'tributo affettivo' quando a essere richiamate sono le memorie condivise così come i ricordi più personali e nostalgici delle persone.

Tutti noi teniamo ancora nella memoria un esempio di 'tributo affettivo' a un grande personaggio come il Mahatma Gandhi. Si tratta di uno spot Telecom girato da Spike Lee nel 2004, in cui si mostra il Mahatma che, dopo essere entrato in una capanna, comincia a parlare di fronte ad una webcam. Fortemente connotate da una musica di atmosfera particolarmente enfatica (il brano $\mathrm{Sa}$ crifice di Lisa Gerrard e Peter Burke), le sue immagini e le sue parole arrivano in megaschermi, schermi di televisori e telefonini sparsi in luoghi diversi del mondo. Lo spot si conclude con la scritta: "Se avesse potuto comunicare così, oggi che mondo sarebbe?" . In questo spot la partecipazione emotiva richiesta allo spettatore gioca sulle immagini di repertorio, sapientemente montate e alternate a quelle degli attori che mostrano il loro coinvolgimento passionale ed emotivo, tanto che passano quasi in secondo piano le parole stesse di Gandhi, tratte da un discorso tenuto il 2 aprile 1947 alla Conferenza interasiatica di fronte a 20.000 persone; un riferimento storico specifico che però, in questo caso, la marca tende a sfruttare più per la sua valenza emotiva e rammemorativa piuttosto che per il suo specifico valore contenutistico.

Già solo questo esempio ci fa capire come la pubblicità si muova completamente in questo ambito di remake, che si colloca a metà tra replica, citazione, riferimento, specialmente se si ha un intento non solo rammemorativo, come nel caso di Telecom, ma anche maggiormente commemorativo. Ed è proprio in questo caso che la dinamica interpretativa comporta l'appropriazione di testi collettivi che possono essere continuamente configurabili da un nuovo e specifico punto di vista. Sono testi sondabili caso per caso perché la loro efficacia co-

7 Per un'approfondita analisi di questo spot cfr. Traini 2005 e 2008, dove l'autore sottolinea i vari aspetti di «partecipazione emotiva» che lo spot veicola e a cui lo spettatore è chiamato ad aderire. 
Cinzia Bianchi | Pubblicità e memoria mediale: appunti in itinere

municativa si basa sulla ricerca di effetti intertestuali localizzati, a volte anche ironici, che sono necessari a richiamare l'attenzione del fruitore.

Ci sono alcuni esempi su cui ci soffermeremo, sebbene il fenomeno sia più ampio e facilmente rilevabile da ognuno di noi nella veste di fruitore di pubblicità ${ }^{\text {. }}$

Il primo esempio commemorativo che vogliamo richiamare è uno spot della Barilla del 2008, uscito in occasione dei 132 anni della fondazione dell'azienda, la cui versione completa di 132 secondi (lo slogan della campagna era infatti: "132 anni, in 132 secondi") ha avuto una diffusione soprattutto legata a Internet. Si tratta di una ricostruzione lineare della storia aziendale a partire dal 1877 , in cui la messa in scena piuttosto patinata rientra completamente nella filosofia pubblicitaria di Barilla. Durante questo racconto ci sono due richiami rammemorativi piuttosto interessanti per il nostro discorso: il primo riguarda i riferimenti specifici ad eventi storici, come il primo allunaggio del 1969, la caduta del muro di Berlino nel 1989 che ci vengono mostrati attraverso i media, le immagini televisive e la prima pagina di un giornale, nel contesto familiare delle cucine coeve all'epoca dell'evento mostrato; il secondo riguarda le moltissime indicazioni su oggetti, consumi e mode vestimentarie, di arredamento, colori e così via dei vari periodi richiamati. In questo caso la storia dell'azienda Barilla attraversa la nostra storia e la nostra memoria collettiva adeguandosi ai tempi, in una continuità di immagini e di intenti.

È questa una prima modalità di rammemorare eventi e usi comuni, che possiamo ritrovare anche nel recente spot di Edison per i suoi 130 anni. Sebbene più ironico, quest'ultimo spot ricalca le modalità di richiami al contesto storico, a costumi e comportamenti specifici in una proiezione verso il futuro della narrazione vincente, propria di coloro che, con lungimiranza, hanno previsto ciò che poi sarebbe accaduto (l'utilizzo capillare dell'elettricità così come la valorizzazione della pasta come cibo apprezzato in tutto il mondo). In questi casi lo spettatore è chiamato a comprendere l'appropriatezza della storia narrata e a riconoscerne l'indubbio valore.

Diverso è il caso dello spot del 2010 prodotto per i cinquanta anni del Cornetto Algida, un compleanno che i pubblicitari decidono di festeggiare in modo autoreferenziale, ripercorrendo, sempre in modo lineare e cronologico, le principali pubblicità del Cornetto stesso. La strategia complessiva è qui del tutto diversa: c'è infatti un tentativo di coinvolgimento dello spettatore molto più personale, come se in questo susseguirsi di immagini e situazioni ognuno potesse riconoscere il 'proprio' Cornetto Algida, quello della sua adolescenza, dei primi amori, di quando il noto gelato ha prodotto un'emozione intensa, di quelle che non si dimenticano per tutta la vita... Per ottenere tale effetto la strategia si basa essenzialmente sull'intertestualità: citazioni e riferimenti tutti interni al discorso della marca e alla comunicazione pubblicitaria stessa.

Sebbene la ricostruzione lineare della storia aziendale, della marca o del singolo prodotto sia la modalità più utilizzata nella pubblicità commemorativa,

8 Tale fenomeno di remake/remix pubblicitario può essere anche collegato all'ampia diffusione, specialmente tra i giovanissimi, di un gusto retrò che riguarda molte pratiche e sfere di consumo e molti tipi di audiovisivi, così come viene approfondito da Daniela Panosetti e Maria Pia Pozzato nel libro: Passione vintage (2013). 
Cinzia Bianchi | Pubblicità e memoria mediale: appunti in itinere

ci sono tuttavia esempi di anniversari o lanci di restyling di prodotto più impegnativi dal punto di vista dell'investimento formale e intertestuale e tale investimento si misura innanzitutto a partire dalla loro configurazione più rizomatica, che va a pescare immagini e situazioni non cronologicamente ordinate.

Un caso piuttosto interessante di questo tipo è lo spot della Nuova FIAT500, mandato in onda sulle reti televisive italiane e sui maggiori siti Internet a partire dal 4 luglio 2007, a cinquanta anni esatti dal debutto della prima Cinquecento che, ci raccontano le cronache dell'epoca, fu festeggiato a Torino con una sfilata di ben 150 vetture. Anche nel 2007 l'evento è stato caratterizzato da ingenti manifestazioni a Torino, oltreché nelle piazze centrali di Milano, Roma e altre città, in concomitanza con il lancio ufficiale.

Lo spot racconta avvenimenti salienti della recente storia italiana, non trascurando avvenimenti tragici del nostro passato ma gioendo delle belle avventure e delle belle persone del nostro caro paese; il tutto filtrato idealmente dallo sguardo di Totò Cascio, protagonista da bambino del celeberrimo film di Giuseppe Tornatore "Nuovo Cinema Paradiso" del 1988. Si tratta di uno spot in parte istituzionale ma anche di prodotto, che gioca anzi sulle possibili sinergie tra marca/prodotto e storia nazionale; cerca cioè, nel suo intento rammemorativo e commemorativo, di identificare la storia dell'industria automobilistica Fiat con la storia italiana.

Tale identificazione è perseguita attraverso tre mosse narrative: il testo dello spot, letto dalla voce fuori campo di Ricky Tognazzi, in cui l'opposizione tra giusto/sbagliato e bene/male indica, con un sincretismo verbo-visivo, ciò di cui essere orgogliosi e fieri e ciò di cui rammaricarsi o per cui provare dolore; $\mathrm{i}$ brani del film di Tornatore che fanno da cornice emotivo/passionale alla parte visiva; il montaggio di immagini scelte da Mauro Vallinotto (fotografo e allora giornalista del quotidiano La Stampa) che ritraggono grandi personaggi, avvenimenti o tragedie del nostro passato, ma che sfruttano al contempo anche alcuni stilemi della pubblicità con bambini, belle donne, famiglie felici. Gli ultimi fotogrammi riprendono invece l'evoluzione del marchio FIAT dall'inizio fino agli ultimi restyling. Come direbbe Isabella Pezzini (2002: 7) le inquadrature nel loro insieme "danno luogo a una fitta rete di rinvii fra testi, storie, personaggi, ambienti, una rete satura di circolazione e raccordi di desideri, curiosità, attese e nostalgie", di cui ogni fruitore può beneficiare richiamando memorie individuali o collettive perché sono immagini che ci sono state più volte proposte come caratterizzanti un certo periodo o avvenimenti del nostro passato.

Dal punto di vista semiotico è un testo piuttosto sofisticato, che non si basa sulla linearità temporale (come in altri spot dello stesso genere) ma subordina le opposizioni a un sottostante livello valoriale esplicito, mostrato e letto: bene/ male, giusto/sbagliato, cosa essere e cosa non essere per diventare qualcos'altro, autentici e veri, qualcosa di simile ai grandi personaggi mostrati, ma anche agli stereotipi di molte persone comuni. $\mathrm{E}$ in questo processo si costruisce appunto l'appartenenza a una identità nazionale, di cui fa parte anche la Fiat9 .

9 Per un'analisi approfondita di questo spot e dell'intera campagna Fiat500 (annunci stampa, performance, installazioni ecc.), cfr. Manzo 2010. 


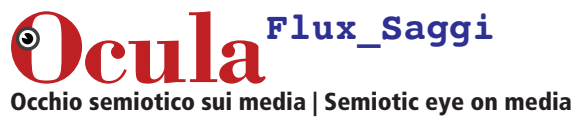

Cinzia Bianchi | Pubblicità e memoria mediale: appunti in itinere

\section{Note conclusive}

Questi pochi esempi ci permettono di concludere questo nostro saggio con alcune riflessioni sui meccanismi generali e particolari della pubblicità.

Ci sembra di aver abbondantemente ribadito quale sia l'importanza dei rimandi intertestuali e dei richiami enciclopedici dal punto di vista sia di chi produce pubblicità sia di chi ne è fruitore. Vogliamo qui sottolineare come nella dinamica produzione/fruizione ogni testo si costituisca sempre come temporaneo punto di approdo del processo interpretativo; ogni testo può quindi a sua volta divenire fonte di ulteriore creatività, così come di ulteriore interpretazione, sempre aperta a nuove o diverse "passeggiate inferenziali" (cfr. in particolare Eco 1994).

Se poi il gioco interpretativo proposto dalla pubblicità si basa sul richiamo ad eventi o passioni del passato, allora la stessa dinamica interpretativa può diventare molto più interessante per uno studioso di cultura di massa perché la rammemorazione diventa quasi una ricerca e una sfida creativa all'individuazione di ciò che ci appartiene come collettività, di ciò che si può considerare veramente identitario della nostra cultura, insomma un livello ampiamente condiviso del sapere comune, difficilmente identificabile in modo così generalizzato. Per questo motivo possiamo rilevare la sporadicità dell'investimento della pubblicità in testi che hanno come fonte insostituibile la memoria collettiva; ci si investe solo in casi eccezionali, come nelle campagne commemorative nelle quali si scommette sul valore del messaggio da trasmettere e sulla temporanea sospensione del discorso di marca. Si tratta di un impegno intenso ed eticamente apprezzabile ma 'a termine', per poi tornare alla solita filosofia dell'estemporaneità tipica del discorso pubblicitario.

\section{Bibliografia}

Bianchi C.

2005 Spot. Analisi semiotica dell'audiovisivo pubblicitario, Roma, Carocci.

$\mathrm{Ead}$

2008 "Raccontare la storia. Fiction e docufiction, tra ciotole di latte, antenne e buste della spesa", in Pozzato M. P., Grignaffini G. (a cura di), Mondi seriali. Percorsi semiotici nella fiction, Milano, RTI LINK Ricerca, pp. 227- 243.

Bianchi C., Ragonese R.

2013 L'annuncio pubblicitario, Roma, Carocci.

Demaria C.

2006 Semiotica e memoria. Analisi del post-conflitto, Roma, Carocci.

Dusi N., Spaziante L. (a cura di)

2006 Remix-Remake: pratiche di replicabilità, Roma, Meltemi.

Eco U., 1979, Lector in fabula, Milano, Bompiani.

1984 Semiotica e filosofia del linguaggio, Torino, Einaudi.

1990 I limiti dell'interpretazione, Milano, Bompiani.

1994 Sei passeggiate nei boschi narrativi, Milano, Bompiani. 


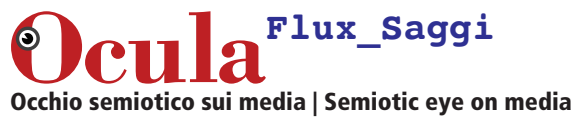

Cinzia Bianchi | Pubblicità e memoria mediale: appunti in itinere

Esposito E.

2001 La memoria sociale. Mezzi di comunicazione e modi di dimenticare, Bari, Laterza.

Ferraro G.

2000 Uno sguardo in camera, Milano, Cooperativa Libraria Iulm.

Grasso A. (a cura di)

2006 Fare storia con la televisione. L'immagine come fonte, evento, memoria, Milano, Vita e pensiero.

Lotman J.

1985 La semiosfera. L'asimmetria e il dialogo nelle strutture pensanti, Marsilio, Venezia.

Lotman J., Uspenski B.

1975 Tipologia della cultura, Milano, Bompiani.

Manzo L.

2010 "La nuova Fiat appartiene a tutti noi. Analisi semiotica della campagna di lancio pubblicitario della nuova Fiat 5oo", in Ocula- FluxSaggi, vol 11, pp. 1-33. http://www.ocula.it/files/Manzo_OculaFlux_saggi_[3,536.455M].pdf

Panosetti D., Pozzato M. P.

2013 Passione vintage, Roma, Carocci.

Pezzini I. (a cura di)

2002 Trailer, spot, clip, siti, banner. Le forme brevi della comunicazione audiovisiva, Roma, Meltemi.

Ricoeur P.

1998 Das Rätsel der Vergangenheit. Erinnern - Vergessen - Verzeihen, Göttingen: Wallstein (tr. it. Ricordare, dimenticare, perdonare, Bologna, il Mulino, 2004).

Silverstone R.

1999 Why study the media?, London, Sage (tr. it. Perché studiare i media?, Bologna, il Mulino, 2002).

Treleani M.

2014 Mémoires audiovisuelles. Les archives en ligne ont-elles un sens ?, Montréal (CA), Les presses de l'Université de Montréal.

Traini S.

2005 "Estesie di marca. Aspetti sensoriali e somatici della brand communication", in C. Bianchi e S. Traini (a cura di) "Sguardi semiotici sulla pubblicità", Ocula, vol. 6, n. 6, pp. 1-20. http://www.ocula.it/files/trainio6 [158,873Kb].pdf

2008 Semiotica della comunicazione pubblicitaria, Milano, Bompiani.

Cinzia Bianchi è ricercatrice di semiotica presso la Facoltà di Scienze della Comunicazione e dell'Economia dell'Università di Modena e Reggio Emilia. Ha lavorato presso diverse università italiane (Bologna, Milano-IULM, Università Statale di Milano). Oltre a numerosi saggi riguardanti la semiotica e la cultura di massa, ha pubblicato $\mathrm{Su} \mathrm{Fer-}$ ruccio-Rossi-Landi (Esi, 1995), Spot: analisi semiotica dell'audiovisivo pubblicitario" (Carocci, 2005), (con altri) Spettri del potere (Meltemi, 2002) e L'annuncio pubblicitario (Carocci, 2013). Ha curato un numero della rivista internazionale "Semiotica" dedicato al pensiero di Umberto Eco (vol. 2015, issue 206). 